\title{
Diagnóstico y escenarios de peligro por inundación fluvial en Ciudad Insurgentes, Baja California Sur, México
}

\author{
Diagnosis and potential dangers of river flooding in Ciudad Insurgentes, \\ Baja California Sur, Mexico \\ \aúl Chávez López*凶, Eric Avilés Canett* \\ Chávez López, S., \& Avilés Canett, E. (2017). Diagnóstico y escenarios de peligro por \\ inundación fluvial en Ciudad Insurgentes, Baja California Sur, México. Investigación y \\ Ciencia de la Universidad Autónoma de Aguascalientes, 25(72), 62-74.
}

\section{RESUMEN}

La elaboración de mapas de peligro se efectuó identificando eventos históricos asociados con inundaciones y la estimación de su periodo de retorno. Los aspectos geomorfológicos e hidrográficos de la cuenca del arroyo Insurgentes no son factores que durante lluvias intensas incrementen el riesgo de inundaciones; estas se presentan como respuesta a la pérdida de área de escurrimiento, debido al incremento de la mancha urbana, que se incorpora como factor inducido. De los resultados del modelaje se encontró que la inundación producida al paso del huracán Jimena en 2009 fue similar a la estimada para un periodo de retorno de 10 años, con un área inundable de $1.2 \mathrm{~km}^{2}$, la cual puede llegar a incrementarse en $94 \%$ para un periodo de retorno de 1000 años, por lo cual se infiere que en el poblado de Ciudad Insurgentes durante la temporada de huracanes se pueden alcanzar situaciones críticas de inundación.

\section{ABSTRACT}

River flood risk maps were generated based on the identification of historic events associated with

Palabras clave: cuenca; geomorfología; huracán; riesgo inducido; inundaciones; periodos de retorno.

Keywords: basin; geomorphology; hurricane; induced risk; flooding; return period.

Recibido: 8 de marzo de 2017, aceptado: 15 de septiembre de 2017

* Programa de Planeación y Conservación Ambiental, Centro de Investigaciones Biológicas del Noroeste, S. C. Instituto Politécnico Nacional 195, Playa Palo Santa Rita Sur, C. P. 23096, La Paz, B. C. S., México. Correo electrónico: schavez04@cibnor.mx; ericavilescanett@gmail.com

$凶$

rreo electrónico: schavez04@
Autor para correspondencia flooding and the estimation of their return period. The geomorphological and hydrographic aspects of the Insurgentes stream basin are not factors that during intense rains increase the risk to floods in the town for natural effect; these comes like answer to the loss of area of glide, due to the increment of the urban area, that incorporate like induced factor lacking of territorial planning in the environment geomorphological of the basin. Based on the modelling results, the flood produced in the wake of Hurricane Jimena in 2009 was similar to that estimated for a 10-year period, with a flood plain of $1.2 \mathrm{~km}^{2}$; the flood plain could increase by $94 \%$ for a 1,000 year return period. In conclusion, the population of Ciudad Insurgentes faces a critical flood risk during the hurricane season.

\section{INTRODUCCIÓN}

Ciudad Insurgentes se fundó en 1953 como una colonia agrícola, según el Instituto Nacional para el Federalismo (INAFED, 1987), destacando en el estado de Baja California Sur desde la década de los sesenta a la de los ochenta del siglo pasado por su producción de algodón y trigo, la cual colapsó debido a la sobreexplotación de sus mantos acuíferos (INEGI, 1996; Troyo-Diéguez et al., 2010). Dicho colapso se vio reflejado en una reducción en su población entre los años de 1990 y 2000, de 8,463 a 7,654 habitantes, respectivamente; para el año 2015 en el sitio en estudio la población aumentó a 10,767 habitantes; cuya economía se encuentran recuperando gracias al auge que está tomando la agricultura en la producción de hortalizas (Gobierno del estado de Baja California Sur, 2015). Sin embargo, su economía también se ha visto afectada por inundaciones producto de lluvias intensas asociadas 


\section{IIVESTIGAGIÓn Y CUERCIA DE LA UกIVERSIDAD AUTÓOOOMA DE RGUASCHLIETTES}

63

a huracanes. La alta incidencia de estos en la región hace que los fenómenos meteorológicos sean la principal amenaza a la que se encuentra expuesta la población de Ciudad Insurgentes, al ocasionar daños a viviendas, negocios, servicios públicos, así como pérdidas en la producción agrícola, de la cual depende la mayor parte de la población, como encontró el Centro Nacional de Prevención de Desastres (CENAPRED, 2009).

Los primeros antecedentes de inundaciones en Ciudad Insurgentes son de octubre de 2008, a partir de ahí han sido recurrentes; sin embargo, la información referente a inundaciones tanto a nivel local como regional es escasa y se encuentra dispersa en dependencias de orden municipal como las oficinas del Sistema de Agua Potable y Alcantarillado (SAPA) y el Comité de Protección Civil del Municipio de Comondú. Aunado a la escasa información, así como al lento pero constante crecimiento demográfico en Ciudad Insurgentes y a la importancia agrícola y económica que tiene para el estado de Baja California Sur, se justifica elaborar el diagnóstico de riesgo a inundaciones, partiendo de los aspectos geomorfológicos y patrón fluvial de la cuenca. Estos son aspectos que Gutiérrez Elorza (2008) refiere como importantes a considerar para el diagnóstico de inundaciones fluviales, en donde la combinación de su forma con el sistema de drenaje influye en los máximos o picos de la inundación; así como el modelar escenarios de peligro por inundaciones fluviales para diferentes periodos de retorno. Para justificar el presente trabajo los autores plantean a manera de hipótesis que los problemas de inundación en Ciudad Insurgentes se presentan como un riesgo inducido debido al incremento del área urbana carente de planeación y ordenamiento. Aspectos que aun cuando en el presente trabajo se presentan como una realidad local, es posible afirmar que situaciones muy parecidas se presentan en todo México.

\section{Área de estudio}

La cuenca del arroyo Insurgentes corresponde a la región hidrológica RH03, Baja California Sur-Oeste, Magdalena (INEGI, 1996), en cuya porción media se encuentra el poblado de Ciudad Insurgentes (figura 1).

El clima en la región es seco semicálido, con temperaturas mensuales más altas y mayores precipitaciones acumuladas entre los meses de agosto y septiembre, de 29 a $35^{\circ} \mathrm{C}$ y de 50 a $60 \mathrm{~mm}$, respecti-

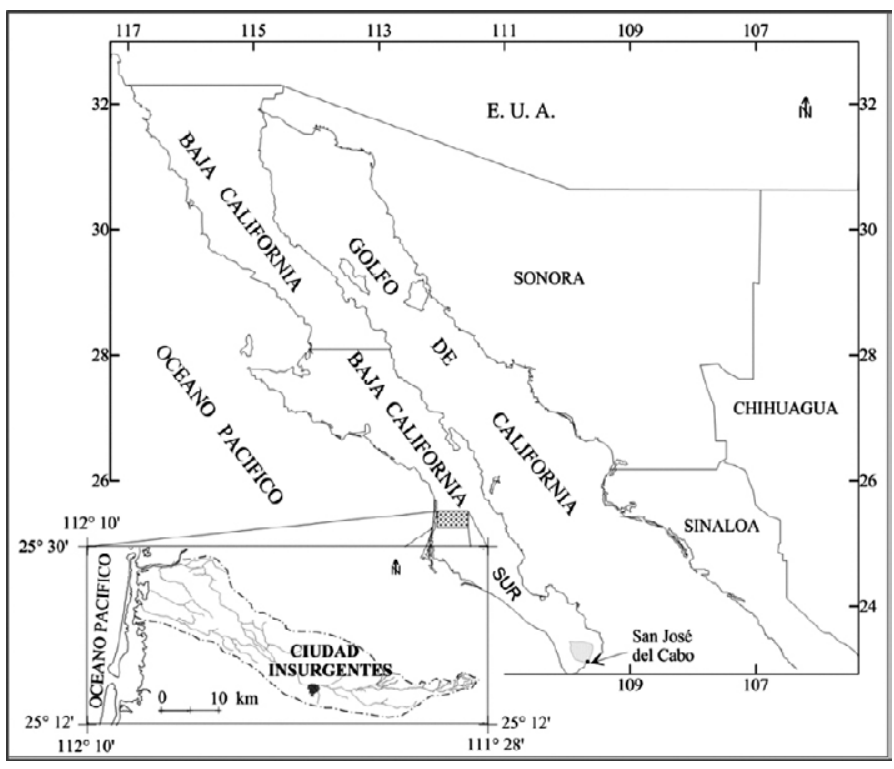

Figura 1. Localización del área en estudio. Elaboración propia.

vamente (INEGI, 1996). Los aspectos climáticos se reflejan en la vegetación, con predominancia de cactáceas como son el cardón (Pachycereus pringlei), pitahaya agria (Machaerocereus qummosus), pitahaya dulce (Stenocereus thurberi), entre otras, que se encuentran en la mayor parte de la región, exceptuando las áreas deforestadas para uso agrícola (Arriaga, Coria, \& Bastida-Zavala, 1992; INEGI, 1996). Los suelos predominantes son arenosol, caracterizados por una textura gruesa, baja capacidad de retención de nutrientes y muy permeables; si se riegan pueden soportar una gran variedad de cultivos (Silva Machorro, 1978). Desde el punto de vista fisiográfico INEGI (1996) ubica al área de estudio en la subprovincia Discontinuidad Llanos de Magdalena, la cual coincide con la subprovincia geológica que López Ramos (1979) denomina como Llanos Iray-Magdalena, integrada por depósitos Cuaternarios derivados de la sierra La Giganta.

Los aspectos descritos ubican a la cuenca del arroyo Insurgentes y al poblado del mismo nombre en una llanura caracterizada por su aridez, por lo que los arroyos son de régimen intermitente; aspectos que no son condicionantes para ocasionar inundaciones. Se han producido en el poblado como inundaciones súbitas, debido a fenómenos meteorológicos, que ocasionan lluvias intensas y cuyos aspectos se retoman con más detalle en el apartado de resultados. 
Administration (NOAA, 2016). Con la base de datos de lluvias máximas y empleando el método Gumbel El trabajo sigue la metodología del CENAPRED (CENAPRED, 2013; Ramos Radilla, 2006; Salas Salinas, 2011) para modelar los escenarios de peligro por inundaciones fluviales para diferentes periodos de retorno; lo cual consiste en determinar el peligro mediante la delimitación de inundaciones históricas e identificación del evento asociado a esta y la estimación de su periodo de retorno (CENAPRED, 2013).

Como plataforma de trabajo se empleó el sistema de información geográfica Arc-GIS ver. 10.2, apoyados con el programa Surfer versión 8 para la elaboración de figuras. Para las bases de datos de precipitación e incidencia de huracanes, así como elaboración de gráficos, se empleó la hoja de cálculo de Microsoft Office Excel 2010. El mapa base se elaboró con las cartas topográficas vectoriales del Instituto Nacional de Estadística y Geografía (INEGI, 1998, 2000): G12C37 (Ciudad Insurgentes) y G12C47 (Benito Juárez), escala 1:50,000; con cuya información a su vez se generó el modelo digital de elevación (MDE) con resolución de $15 \mathrm{~m}$, con el cual se trazó el parteaguas de la cuenca del arroyo Insurgentes y se obtuvieron sus aspectos morfométricos y patrón de drenaje fluvial, tipificado de acuerdo con los órdenes de corrientes de Horton.

El trabajo de campo se efectuó del 9 al 14 de marzo y del 29 de junio al 4 de julio de 2015 y consistió en el reconocimiento de la cuenca y descripción de unidades geomorfológicas, apoyados con un GPS MobileMapper 6 de la marca Magellan; así como recolección de información de inundaciones históricas y bibliografía, con funcionarios del Municipio de Comondú, Protección Civil y pobladores de Ciudad Insurgentes, la cual se empleó para la delimitación de inundaciones históricas y para validar el modelaje realizado para los escenarios de peligro a inundaciones fluviales.

El análisis de precipitaciones se realizó con datos por día para los años comprendidos desde 1940 hasta 2012, de las estaciones meteorológicas que se muestran en la figura 3, cuya información fue proporcionada por la Subdirección de Asistencia Técnica Operativa de la Comisión Nacional del Agua, Baja California Sur. La incidencia de huracanes se obtuvo con la depuración de la base de datos de las estaciones meteorológicas antes referidas, reportadas como lluvias máximas y complementadas con información de la National Oceanic Atmospheric $5,10,25,50,100,200,500$ y 1000 años, corobora 2 $25,50,100,200,500$ y 1000 años, corroboradas con los mapas de isoyetas de Ramos Radilla (2006). Los periodos de retorno y el método empleado para la elaboración de mapas de isoyetas fueron seleccionados con la finalidad de que los resultados del modelaje fueran compatibles con los de los Atlas de Riesgos Municipales y Estatales, según la metodología del CENAPRED (CENAPRED, 2013; Ramos Radilla, 2006; Salas Salinas, 2011).

Como parte de dicho método se empleó la información generada en los apartados de geomorfología, hidrología y precipitaciones e incidencia de huracanes; así como el Simulador de Flujos de Agua de Cuencas Hidrográficas SIATL del INEGI (2016b); de donde se obtuvo el coeficiente de escurrimiento de la cuenca y los cálculos de caudales del arroyo Insurgentes para los diferentes periodos de retorno estimados. Para el modelaje de escenarios de peligro se empleó el programa HEC-RAS (Hidrologic Engineering Centers River Analysis System), que permite realizar simulaciones hidráulicas unidimensionales.

Para el análisis del aumento poblacional se empleó información de los Censos de Población y Vivienda del (INEGI, 2016a), complementada con datos de la Comisión Nacional del Agua (CONAGUA, 2015) y del Gobierno del estado de Baja California Sur (2015). El incremento del área urbana se analizó mediante la sobreposición de las áreas digitalizadas en fotografía aérea de Ciudad Insurgentes para el año de 1973, R-713 de la zona de vuelo 47A línea 3 de INEGl; para los años de 1978 y 1993 se emplearon las cartas topográficas escala 1:50,000, en formato raster de INEGI, G12C37 y G12C47 segunda edición de 1988 y 2000, elaboradas con fotografías aéreas de 1978 y 1993, respectivamente; para digitalizar el área urbana del año 2010 se emplearon las imágenes pancromáticas SPOT 559299 y 559300 y para el año 2015 se emplearon las cartas topográficas G12C37 y G12C47, escala 1:50,000, en formato vectorial de INEGI con actualización al año 2015.

RESULTADOS

\section{Geomorfología}

En la región de la cuenca donde se ubica Ciudad Insurgentes se distinguen dos unidades geomorfológicas: 1) Llanura aluvial y 2) franja costera (figura 2). 


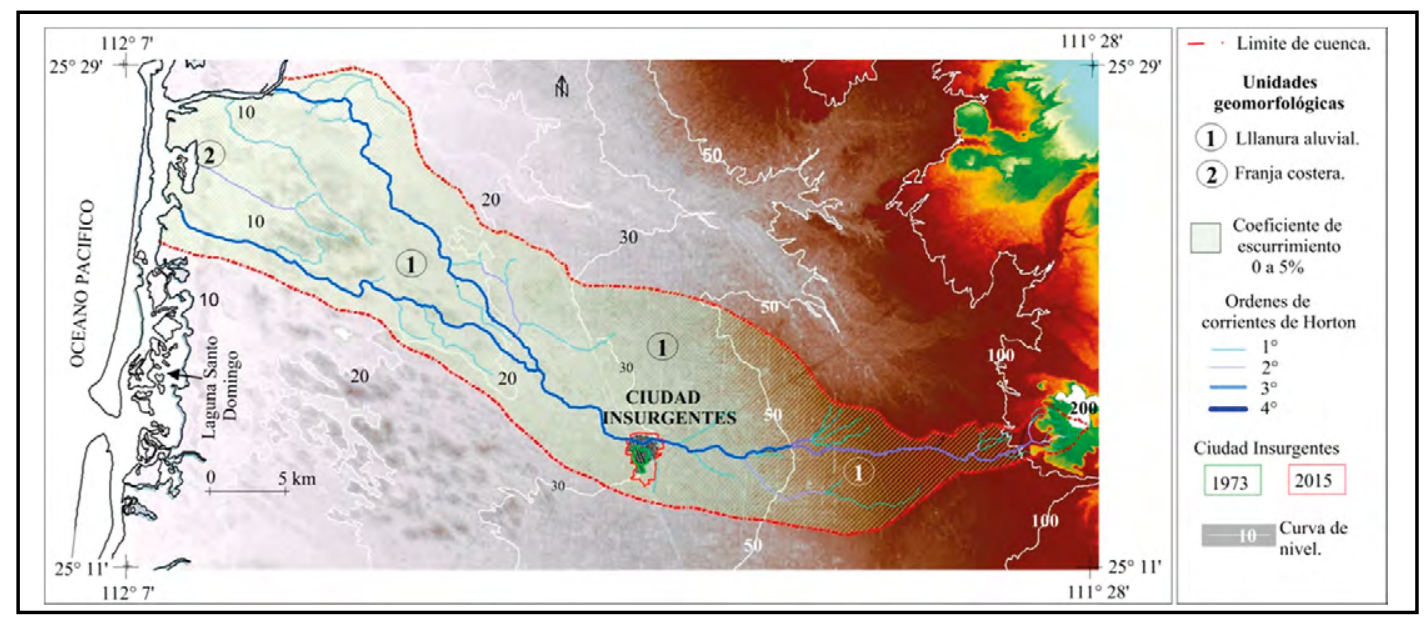

Figura 2. Modelo digital de elevación, unidades geomorfológicas e hidrografía de la cuenca del arroyo Insurgentes. Elaboración propia basada en cartas topográficas vectoriales del INEGI.

1) Llanura aluvial: integra prácticamente la totalidad del área de estudio, y se extiende de oeste a este desde la cota de los 200 m.s.n.m., en donde inicia la zona de bajada con lomeríos (ladera) de la sierra La Giganta, hasta la cota de los 10 m.s.n.m., en donde limita con la franja costera. Presenta una pendiente media de $0.3 \%$ (SIATL), que buza hacia el oeste y se integra por depósitos de aluvión derivados de la sierra La Giganta. Su relieve se ve interrumpido por el drenaje fluvial y el área urbana del poblado de Ciudad Insurgentes ubicado entre los 20 y 30 m.s.n.m., así como por un campo de paleodunas entre la cota de los 10 y 20 m.s.n.m., las cuales integran un paisaje de lomeríos con cimas redondeadas que no sobrepasan los $10 \mathrm{~m}$ de altura.

2) Franja costera: forma parte de la laguna costera Santo Domingo que mantiene comunicación con el Océano Pacífico. Esta unidad geomorfológica integra la desembocadura de la cuenca, con una extensión aproximada de $13.6 \mathrm{~km}$. Su amplitud es variable entre 10 y $50 \mathrm{~m}$, con pendiente suave desde la cota de los 10 m.s.n.m. hasta la línea de costa. Se integra por depósitos litorales Cuaternarios de playas arenosas, así como sedimentos limoarenosos en los pantanos de manglar.

\section{Hidrología superficial}

Dado el relieve plano que caracteriza a la unidad geomorfológica de la llanura aluvial, el parteaguas de la cuenca del arroyo Insurgentes, se traza como del piso del valle (García Gutiérrez, 2003; Lugo Hubp, 1989), lo cual se refleja en un escaso coeficiente de escurrimiento de 0 a $5 \%$. La cuenca es de tipo exorreica, con un perímetro de $170 \mathrm{~km}$ y área de
$632 \mathrm{~km}^{2}$; presenta forma de embudo alargado con dirección sureste-noroeste; su porción más angosta con $2 \mathrm{~km}$ se ubica aguas arriba alcanzando su mayor amplitud en su parte media con $13.8 \mathrm{~km}$, que es en donde se ubica Ciudad Insurgentes y se reduce ligeramente hacia su desembocadura a $13.6 \mathrm{~km}$ (figura 2). De acuerdo con los órdenes de corrientes de Horton, la red hidrográfica es de cuarto orden, integrada por un drenaje dendrítico de escasa densidad y régimen intermitente; el cauce principal es el arroyo Insurgentes con $74 \mathrm{~km}$ de longitud, cuyo recorrido inicia en la zona de bajada de la sierra La Giganta a 200 m.s.n.m. (cabecera de la cuenca). En esta área se integra la mayor densidad de drenaje, que confluye formando un cauce de tercer orden, con un recorrido de $18.56 \mathrm{~km}$ hasta la altura de los 50 m.s.n.m.; a esta altura se integra un tributario de tercer orden, por lo que a partir de esta zona el cauce principal pasa a ser de cuarto orden, lo cual se conserva en el resto de su recorrido, que próximo a la cota de los 20 m.s.n.m. se bifurca en dos ramales que desembocan en la laguna Santo Domingo (figura 2).

De los aspectos físicos de la cuenca destacan su forma de embudo invertido y su escasa pendiente y densidad de drenaje, yaque de acuerdo con Gutiérrez Elorza (2008, p. 330) estos aspectos son importantes a considerar para el diagnóstico de inundaciones fluviales; ya que "una vez que la precipitación llega al suelo, la conversión del flujo dependerá de las características físicas de la cuenca". De acuerdo con los aspectos geomorfológicos e hidrográficos descritos podemos asumir que no son factores que durante lluvias intensas incrementen el riesgo de 


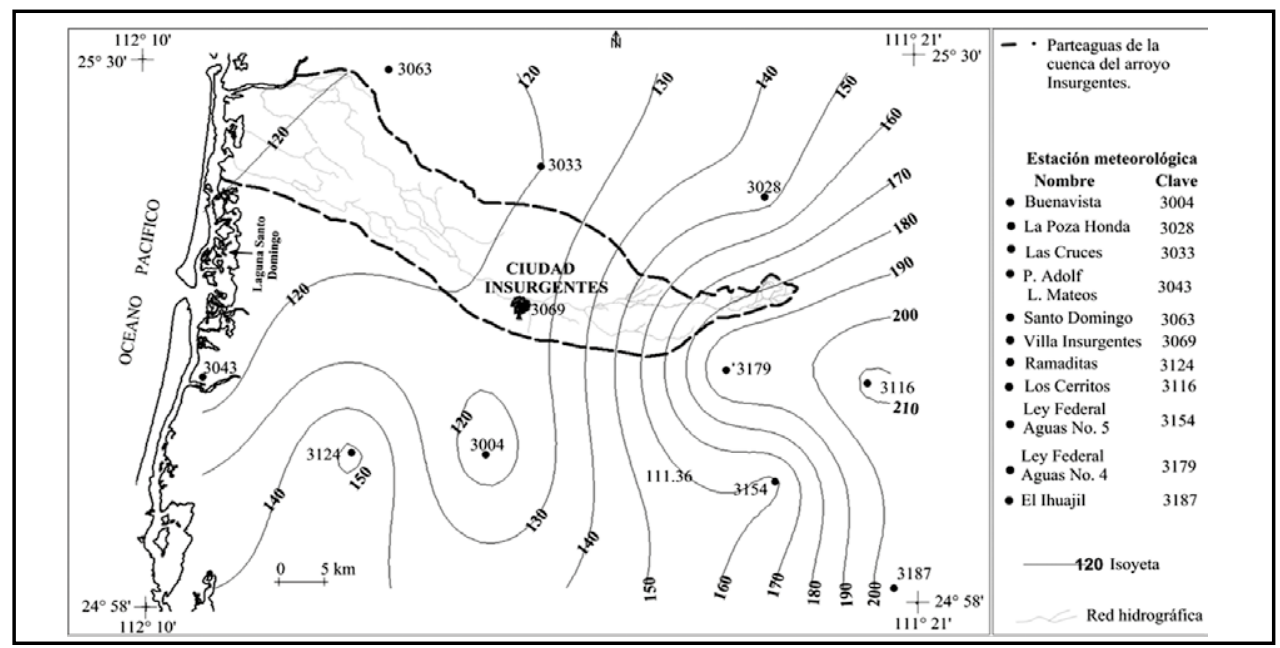

Figura 3. Ubicación de estaciones climatológicas e isoyetas de precipitación media anual de la cuenca del arroyo Insurgentes.

Elaboración propia copn datos de la Subdirección de Asistencia Técnica Operativa de la Comisión Nacional del Agua Baja California Sur, México.

inundaciones en el poblado de Ciudad Insurgentes; como lo son en cuencas como la de San José del Cabo (figura 1), integrada por rocas impermeables, pendientes pronunciadas y forma de embudo, que condiciona una amplia área de captación de lluvia en su cabecera con alta densidad de drenaje, cuyo escurrimiento se reduce hacia su desembocadura en un cauce de sexto orden; aspectos asociados a la cuenca que durante lluvias intensas incrementan de manera natural el riesgo de inundaciones fluviales en el poblado de San José del Cabo (Chávez López \& Mendoza Albañez, 2016).

\section{Precipitaciones e incidencia de huracanes}

En la figura 3 se muestra la distribución de las estaciones climatológicas ubicadas en la cuenca y periferia del arroyo Insurgentes, así como las isoyetas de precipitación media anual. En esta se observa que los valores con precipitación promedio anual superior a los $190 \mathrm{~mm}$ se registran hacia la parte alta de la cuenca, asociados a la zona de bajada de la sierra La Giganta. Ello denota la influencia del gradiente altimétrico de la sierra en la distribución espacial de precipitación, ya que esta se reduce gradualmente conforme se desciende en elevación hacia la franja costera a $120 \mathrm{~mm}$. El área de Ciudad Insurgentes se encuentra en el rango de las isoyetas de 120 a $130 \mathrm{~mm}$ de precipitación media anual.

La variación de precipitación interanual en la región de la cuenca distinguen a los meses de noviembre a febrero como lluvias de invierno, con precipitaciones que no superan los $20 \mathrm{~mm}$; de marzo a junio, la época de estiaje y de julio a octubre los meses más lluviosos pero sin superar en la mayoría de las estaciones los $75 \mathrm{~mm}$ de precipitación. A este último periodo se encuentra asociada la mayor probabilidad de incidencia de huracanes, con énfasis en los meses de agosto y septiembre.

De acuerdo con la revisión de la base de datos de fenómenos hidrometeorológicos, se encontró que en el periodo comprendido desde 1949 hasta 2015, pasaron en un radio de $250 \mathrm{~km}$ a partir de la cuenca del arroyo Insurgentes 52 huracanes (figura 4), promediando 0.8 huracanes por año; lo que ubica

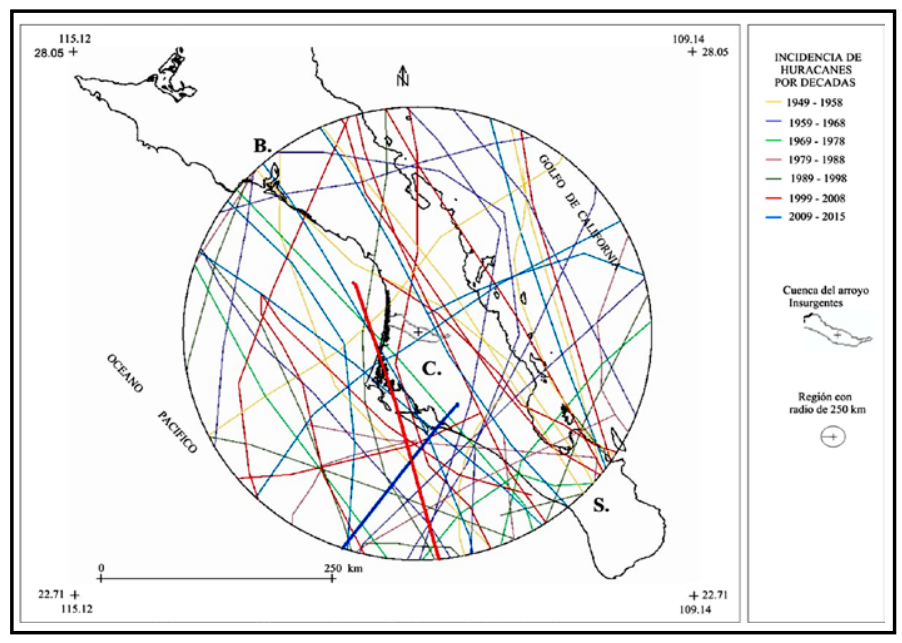

Figura 4. Trayectoria de huracanes que pasaron en un radio de $250 \mathrm{~km}$ a partir de la cuenca del arroyo Insurgentes. Se ordenan en periodos de 10 años y se destacan con línea gruesa en color azul la trayectoria del huracán Norbert en 2008 y en rojo el huracán Jimena en 2009.

Elaboración propia. 


\section{IIVESTIGAGIÓn Y CIERCIA DE LA UחIVERSIDAD AUTÓNOMA DE AGUASCALIERTES}

al área de estudio en una región de alta incidencia a huracanes, en donde estos se convierten en la amenaza principal que condiciona el riesgo a inundaciones.

\section{Incremento del área urbana e inundaciones}

En la figura 5 se muestra la situación que Ciudad Insurgentes presentaba en el año de 1973, la cual se asume como respuesta a la demanda de vivienda, por el aumento poblacional desde su fundación en 1953, con un grupo de 80 personas, la cual pasó a 2,473 habitantes en el año de 1970, cuando el poblado apenas cubría una superficie de $1 \mathrm{~km}^{2}$. De la figura 5 hay que resaltar que el poblado se encontraba a $0.3 \mathrm{~km}$ al sur del arroyo Insurgentes y limitando al oeste con uno de sus tributarios de primer orden, que se origina en la llanura aluvial con un recorrido en dirección sureste-noroeste de 3.6 $\mathrm{km}$. También hay que destacar que entre los años de 1953 y 1973, en el radio de 250 km a partir de la cuenca del arroyo Insurgentes (figura 4), pasaron 15 huracanes sin que ninguno de ellos ocasionara inundaciones en el poblado.

En la tabla 1 se resume información del aumento poblacional e incremento del área urbana de Ciudad Insurgentes (AU) y en la figura 6 se muestra su distribución espacial. El efecto que dicho incremento ha tenido en el entorno geomorfológico de la cuenca se evidencia al comparar las figuras 5 y 6. En esta última figura y teniendo como referencia el polígono que ocupaba el poblado en el año de 1973, se observa que la tendencia de incremento de

Tabla 1

Aumento poblacional e incremento de la mancha urbana en Ciudad Insurgentes de 1953 a 2015

\begin{tabular}{ccc}
\hline Año & Población & $\mathrm{AU}\left(\mathrm{km}^{2}\right)$ \\
\hline 1953 & 80 & \\
1960 & 510 & \\
1970 & 2473 & \\
1973 & & 1.0 \\
1977 & 7466 & \\
1978 & & 1.7 \\
1990 & 8463 & \\
1993 & & 3.6 \\
2000 & 7654 & \\
2010 & 8741 & 4.5 \\
2015 & 10767 & 5.6 \\
\hline
\end{tabular}

Nota: Elaboración propia.

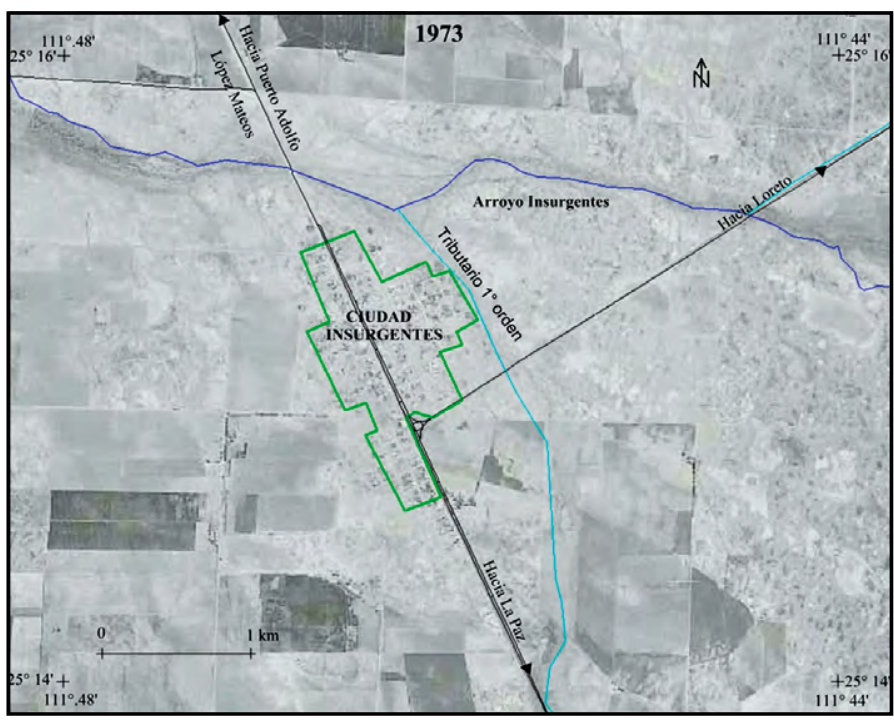

Figura 5. Fotografía aérea del área que ocupaba el poblado de Ciudad Insurgentes en el año de 1973.

Imagen basada en datos de R-713 de la zona de vuelo 47A línea 3 del INEGI.

la mancha urbana hasta el año 2015, se ha dado en su mayor parte hacia el norte y noreste, invadiendo los cauces del arroyo Insurgentes y el de su tributario de primer orden, por lo que actualmente parte de los cauces de dichos arroyos quedan inmersos en el poblado. Es en esta situación de incremento del área urbana que se presentan los primeros antecedentes de inundaciones en octubre de 2008, al paso del huracán Norbert y septiembre de 2009 por Jimena, que en un lapso de $24 \mathrm{~h}$ dejaron en la estación meteorológica Villa Insurgentes (3069) un registro de 59 y $69 \mathrm{~mm}$ de precipitación, respectivamente, superiores a los promedios anuales, lo cual causó el desbordamiento del arroyo Insurgentes.

Según el Sistema de Alcantarillado y Planta de Tratamiento del H. XIII Ayuntamiento de Comondú (2010), el polígono en color azul de la figura 6 indica el área del poblado más afectada por las inundaciones ocurridas al paso de los huracanes antes referidos.

Los habitantes de Ciudad Insurgentes refieren que de los huracanes ocurridos hasta el año 2015, el Jimena en el año 2009 es el que más daños ha causado a viviendas, negocios y servicios públicos, así como pérdidas en la producción agrícola, la cual según el CENAPRED (2009) se estimó en un monto aproximado de 150 millones de pesos. 


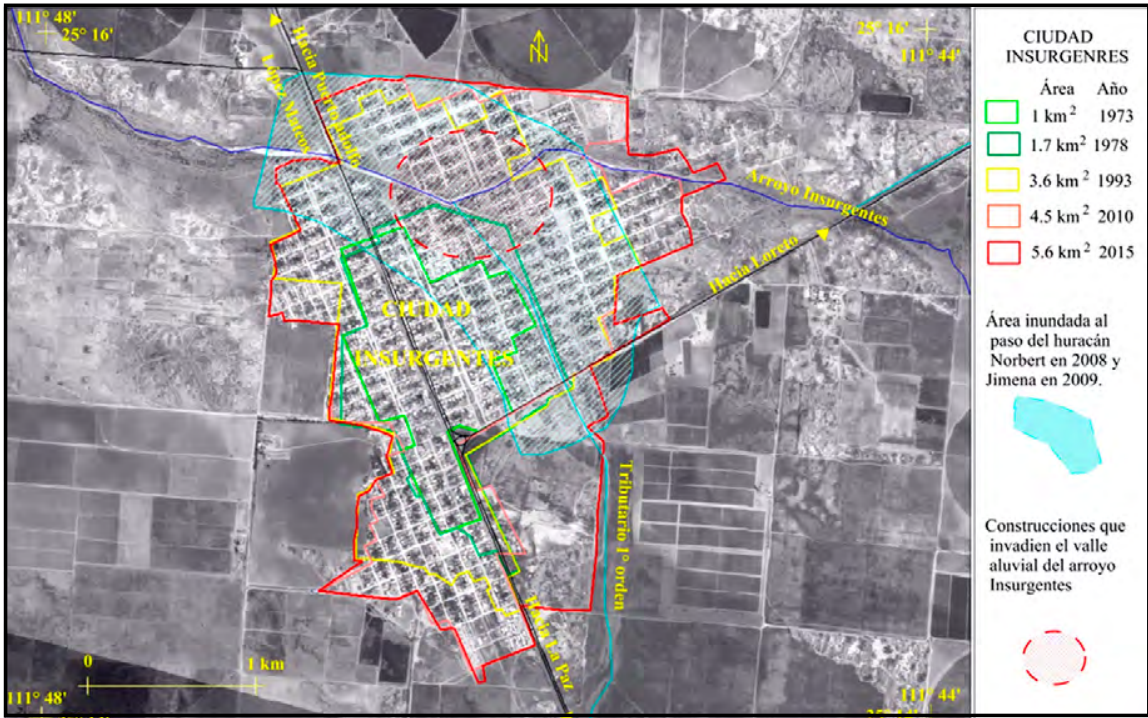

Figura 6. Incremento de la mancha urbana de Ciudad Insurgentes.

Elaboración propia con base en datos de R-713 de la zona de vuelo 47A línea 3 de INEGI, cartas topográficas vectoriales de INEGI $(1988,2000)$ y actualizadas a 2015 , y en fotografías satelitales SPOT 559299 y 559300.

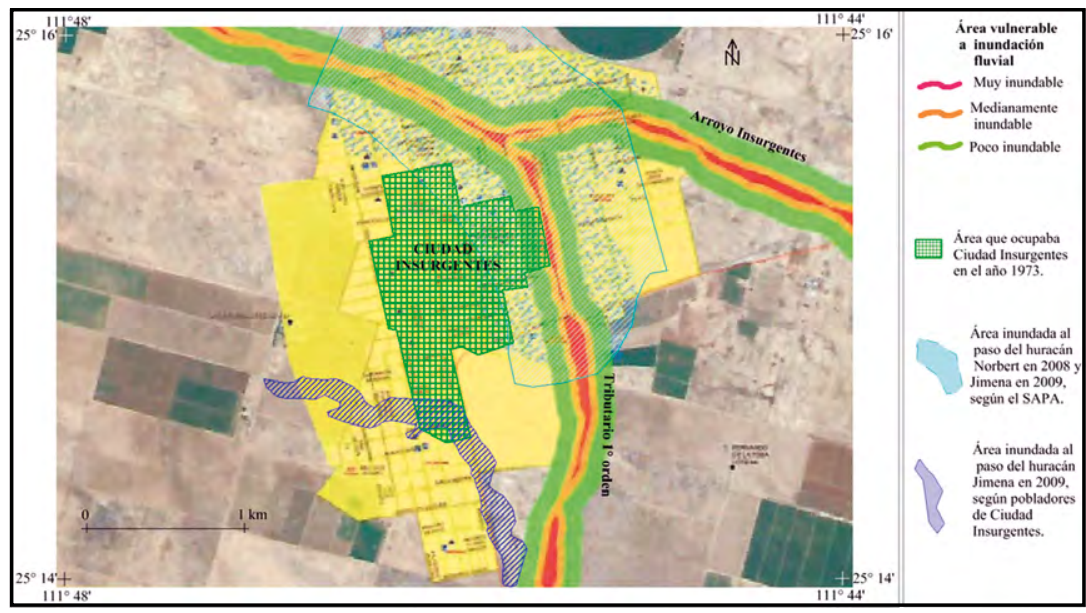

Figura 7. Áreas vulnerables a inundaciones fluviales en Ciudad Insurgentes; el color rojo representa el área muy inundable que corresponde al cauce de los arroyos y el color naranja y verde áreas de mediana a poco inundable, respectivamente, las cuales corresponden a los valles aluviales de los arroyos.

Imagen tomada y modificada del Atlas de Riesgos Naturales del Municipio de Comondú, Baja California Sur, 2011.

Ante dichos problemas de inundaciones es que la Dirección Municipal de Protección Civil del H. XIV Ayuntamiento de Comondú (2011), en un primer intento de elaboración del Atlas de Riesgos Naturales, presenta un mapa de áreas vulnerables a inundaciones fluviales, el cual se muestra en la figura 7. En esta figura se sobrepone un polígono trazado con información registrada con pobladores de Ciudad Insurgentes; quienes refieren que colonias como La Laguna, aun cuando se encuentra retirada de los arroyos, presentó problemas de inundación durante el huracán Jimena, debido a las modificaciones hechas en el terreno durante la construcción de nuevos asentamientos.

De esta información destaca que el problema de inundaciones en Ciudad Insurgentes se presenta como efecto de la poca o nula planificación y ordenamiento de la mancha urbana, por lo que la actividad humana ha cobrado importancia como factor de riesgo inducido que condiciona el problema de estos fenómenos en el poblado (figuras 2 y 6). 


\section{IIVESTIGAGIÓn Y CUERCIA DE LA UחIVERSIDAD AUTÓNOMA DE AGUASCALIERTES}

\section{Escenarios de peligro a inundación}

En la tabla 2 se resumen los resultados del modelado de escenarios de inundación fluvial y en la figura 8 (a) los datos de precipitación registrados en las estaciones climatológicas ubicadas en la periferia y cuenca del arroyo Insurgentes, durante el huracán Jimena en septiembre de 2009, cuya precipitación acumulada alcanzó valores superiores a los $100 \mathrm{~mm}$, similar al de las isoyetas calculadas para un periodo de retorno de 10 años (figura 8 [b]).

Tabla 2

Resumen de resultados del modelado de escenarios de inundación fluvial en Ciudad Insurgentes: 1 Arroyo Insurgentes, 2 Tributario $1^{\circ}$ orden

\begin{tabular}{cccccccc}
\hline $\begin{array}{c}\text { Periodo } \\
(\mathrm{Tr})\end{array}$ & $\begin{array}{c}\text { Precip. } \\
(\mathrm{mm} / 24 \mathrm{~h})\end{array}$ & $\begin{array}{c}\text { Precip. } \\
(\mathrm{mm} / 24 \mathrm{~h})\end{array}$ & $\begin{array}{c}\text { Caudal } \\
(\mathrm{Q}) \\
\left(\mathrm{m}^{3} / \mathrm{s}\right)\end{array}$ & $\begin{array}{c}\text { Caudal } \\
(\mathrm{Q}) \\
\left(\mathrm{m}^{3} / \mathrm{s}\right)\end{array}$ & $\begin{array}{c}\text { Área } \\
\text { inund. } \\
\left(\mathrm{km}^{2}\right)\end{array}$ & $\begin{array}{c}\text { Área } \\
\text { inund. } \\
\left(\mathrm{km}^{2}\right)\end{array}$ & $\begin{array}{c}\text { Área } \\
\text { total } \\
\left(\mathrm{km}^{2}\right)\end{array}$ \\
\hline 2 & 1 & 2 & 1 & 2 & 1 & 2 & \\
\hline 5 & 40 & 40 & 16.45 & 2.76 & 0.69 & 0.59 & $\mathbf{1 . 2 8}$ \\
10 & 83 & 70 & 34.17 & 4.83 & 0.81 & 0.60 & $\mathbf{1 . 4 1}$ \\
25 & 112 & 100 & 46.12 & 6.9 & 0.84 & 0.60 & $\mathbf{1 . 4 4}$ \\
50 & 148 & 130 & 60.93 & 8.98 & 0.84 & 0.61 & $\mathbf{1 . 4 5}$ \\
100 & 201 & 180 & 82.78 & 12.43 & 0.86 & 0.61 & $\mathbf{1 . 4 8}$ \\
200 & 227 & 200 & 93.87 & 13.81 & 0.87 & 0.61 & $\mathbf{1 . 4 9}$ \\
500 & 262 & 230 & 108.32 & 15.89 & 0.89 & 0.62 & $\mathbf{1 . 5 1}$ \\
1000 & 289 & 260 & 119 & 17.96 & 0.89 & 0.62 & $\mathbf{1 . 5 1}$ \\
\hline
\end{tabular}

Nota: Elaboración propia.

En la figura 9 se muestra la simulación de inundación fluvial para el periodo de retorno de 10 años, y se sobreponen las áreas que ocupaban Ciudad Insurgentes en 1973 y en 2015, así como el polígono que destaca el área que según el H. XIII Ayuntamiento de Comondú (2010) fue afectada por la inundación durante los huracanes Norbert y Jimena. En este escenario de inundación la crecida de corriente en el arroyo Insurgentes y su tributario de primer orden superan por $0.4 \mathrm{~m}$ el cauce de los arroyos, generando un área inundable total de 1.44 $\mathrm{km}^{2}$, con un ancho promedio de afectación de 200 $\mathrm{m}$, lo cual representa por lo menos una cuadra de distancia hacia ambos extremos de los cauces de los arroyos. En este contexto las calles en Ciudad Insurgentes, a falta de drenaje pluvial, se incorporan como parte del entorno geomorfológico, formando una intrincada red de drenaje fluvial en donde el agua corre por las calles y las edificaciones actúan como diques, retardando el flujo y la salida de agua hacia la parte baja de la cuenca, inundando así gran parte del poblado.

Los resultados del modelaje para los escenarios de inundación fluvial de los periodos de retorno de $2,5,25,50,100,200,500$ y 1000 años vistos en

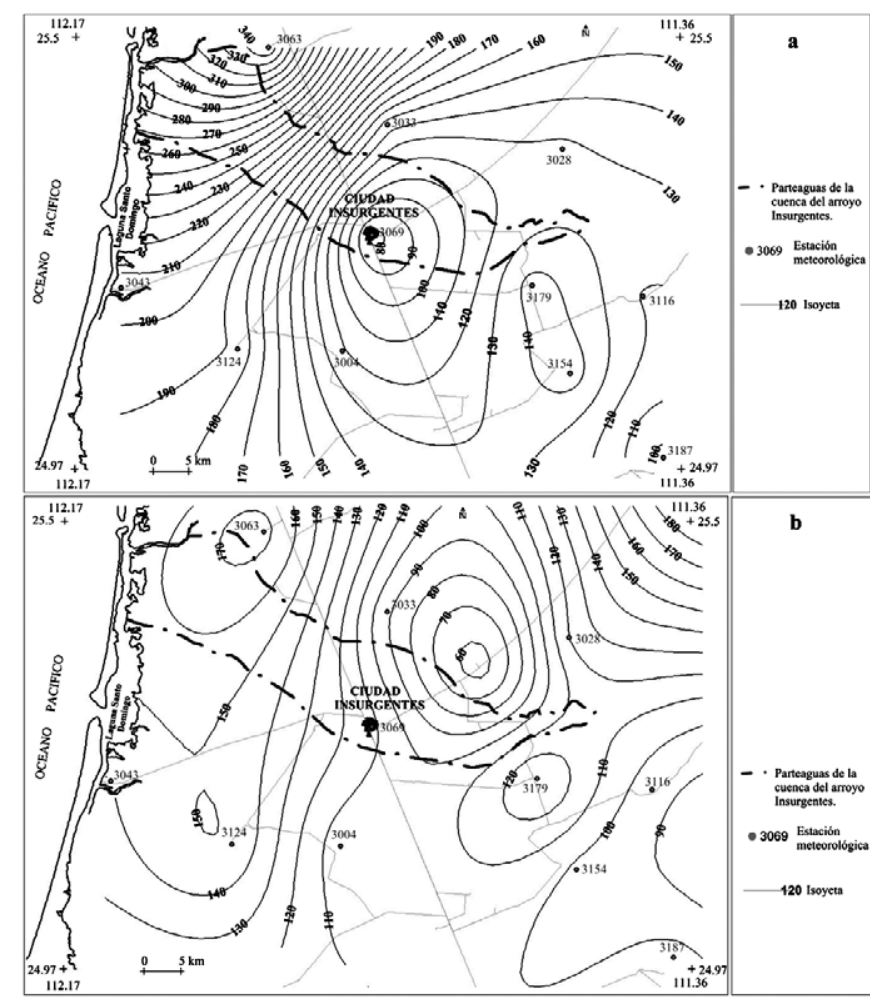

Figura 8. a) Isoyetas del registro de precipitación durante el huracán Jimena en septiembre de 2009; b) Isoyetas calculadas empleando el método Gumbel para un periodo de retorno de 10 años. Elaboración propia. 


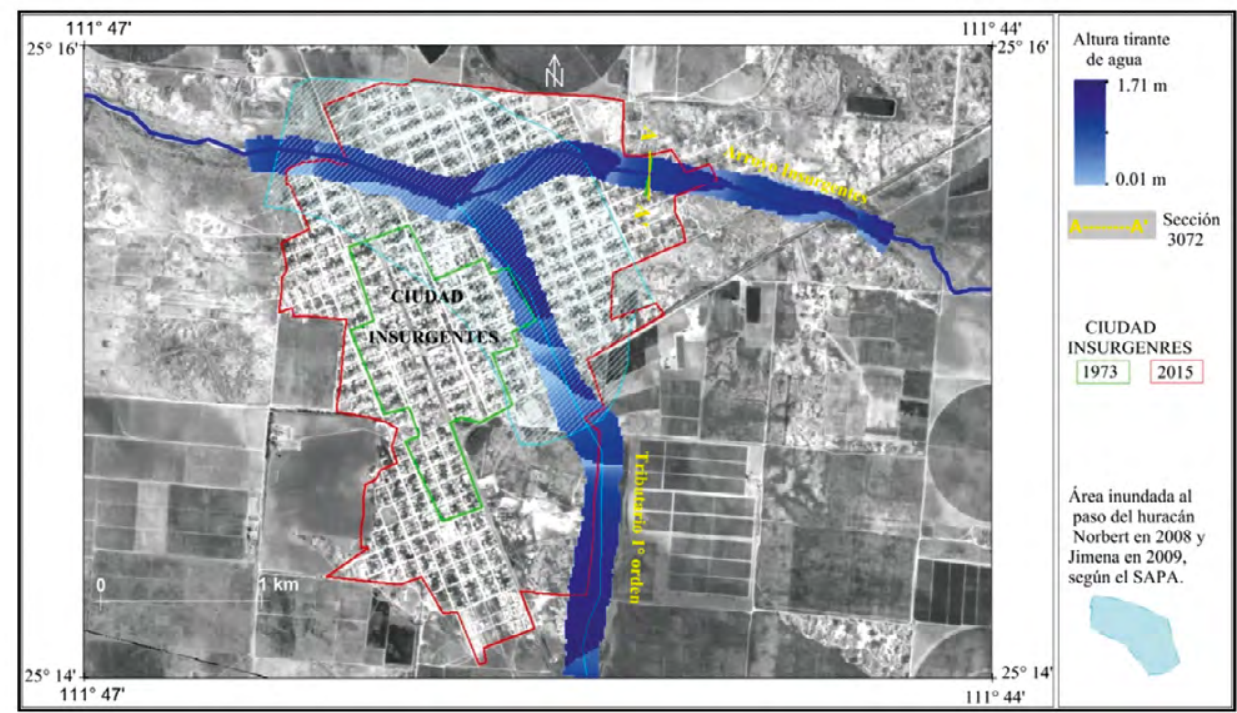

Figura 9. Escenario de inundación fluvial en Ciudad Insurgentes para lluvias estimadas con un periodo de retorno de 10 años.

Elaboración propia basada en R73 de la zona de vuelo 47A línea 3 y en cartas topográficas vectoriales del INEGI.

planta, son muy similares al de 10 años, por lo que para destacar las diferencias de los tirantes de agua generados en los periodos de retorno analizados se emplean los perfiles de la figura 10, cuya ubicación en planta se muestra en la figura 9 como sección 3072 del programa HEC-RAS y en la tabla 3 se resume su información. Cabe mencionar que el tirante de agua y la velocidad que se muestra en los perfiles de la figura 10 varían a lo largo del cauce.

Tabla 3

Resumen de la información de los perfiles de la figura 11, obtenida de la modelación de escenarios de inundación fluvial en Ciudad Insurgentes para diferentes periodos de retorno

\begin{tabular}{ccccc}
\hline $\begin{array}{c}\text { Periodo } \\
\text { de } \\
\text { retorno } \\
(\mathrm{Tr})\end{array}$ & $\begin{array}{c}\text { Profundidad } \\
\text { del cauce } \\
(\mathrm{m})\end{array}$ & $\begin{array}{c}\text { Tirante de } \\
\text { agua }(\mathrm{m})\end{array}$ & $\begin{array}{c}\text { Vel. } \\
\text { Mínima } \\
\text { de } \\
\text { caudal } \\
(\mathrm{m} / \mathrm{s})\end{array}$ & $\begin{array}{c}\text { Vel. } \\
\text { Máxima } \\
\text { de } \\
\text { caudal } \\
(\mathrm{m} / \mathrm{s})\end{array}$ \\
\hline 2 & 1 & 0.90 & 0.2 & 0.8 \\
5 & 1 & 1.25 & 0.4 & 1.0 \\
10 & 1 & 1.45 & 0.6 & 1.2 \\
25 & 1 & 1.60 & 0.4 & 1.2 \\
50 & 1 & 1.75 & 0.6 & 1.4 \\
100 & 1 & 1.85 & 0.6 & 1.4 \\
200 & 1 & 1.95 & 0.6 & 1.4 \\
500 & 1 & 2.10 & 0.8 & 1.6 \\
1000 & 1 & 2.20 & 0.6 & 1.6 \\
\hline
\end{tabular}

Nota: Elaboración propia.
La información de las tablas 2 y 3 y de las figuras 9 y 10 brindan una idea rápida de los posibles escenarios de inundaciones fluviales en Ciudad Insurgentes, como efecto de lluvias esperadas para los periodos de retorno analizados, lo cual se describe como sigue:

La lluvia esperada para un periodo de retorno de 2 años es de $40 \mathrm{~mm} / 24 \mathrm{~h}$ (tabla 2), con lo cual se genera un caudal de $16.5 \mathrm{~m}^{3} / \mathrm{s}$ y un área inundable total de $1.28 \mathrm{~km}^{2}$, con un tirante de agua de $0.90 \mathrm{~m}$, en la zona de la sección 3072 (tabla 3, figura 9), por lo que no supera la altura del cauce del arroyo (figura 10 [a]) y la corriente generada fluye sin desbordar el cauce; sin embargo, en aguas abajo donde el canal del arroyo se amplía actualmente se encuentran construcciones invadiendo el valle aluvial, situación que se destaca en el polígono de color rojo de la figura 6, por lo que aun con un tirante de agua reducido dicha situación para los habitantes de esa zona ya representa un problema de inundación. Con lluvias de $83 \mathrm{~mm}$, esperadas para un periodo de retorno de 5 años, el área total inundable aumenta a $1.41 \mathrm{~km}^{2}$, así como el tirante de agua a $1.25 \mathrm{~m}$ (figura 10 [b]), por lo que su valle aluvial es desbordado con un espejo de agua de $0.25 \mathrm{~m}$, lo que afecta al poblado; tal como ocurrió en la inundación del año 2008 durante las lluvias ocasionadas por el huracán Norbert (figura $11[\mathrm{a}]$ ). 


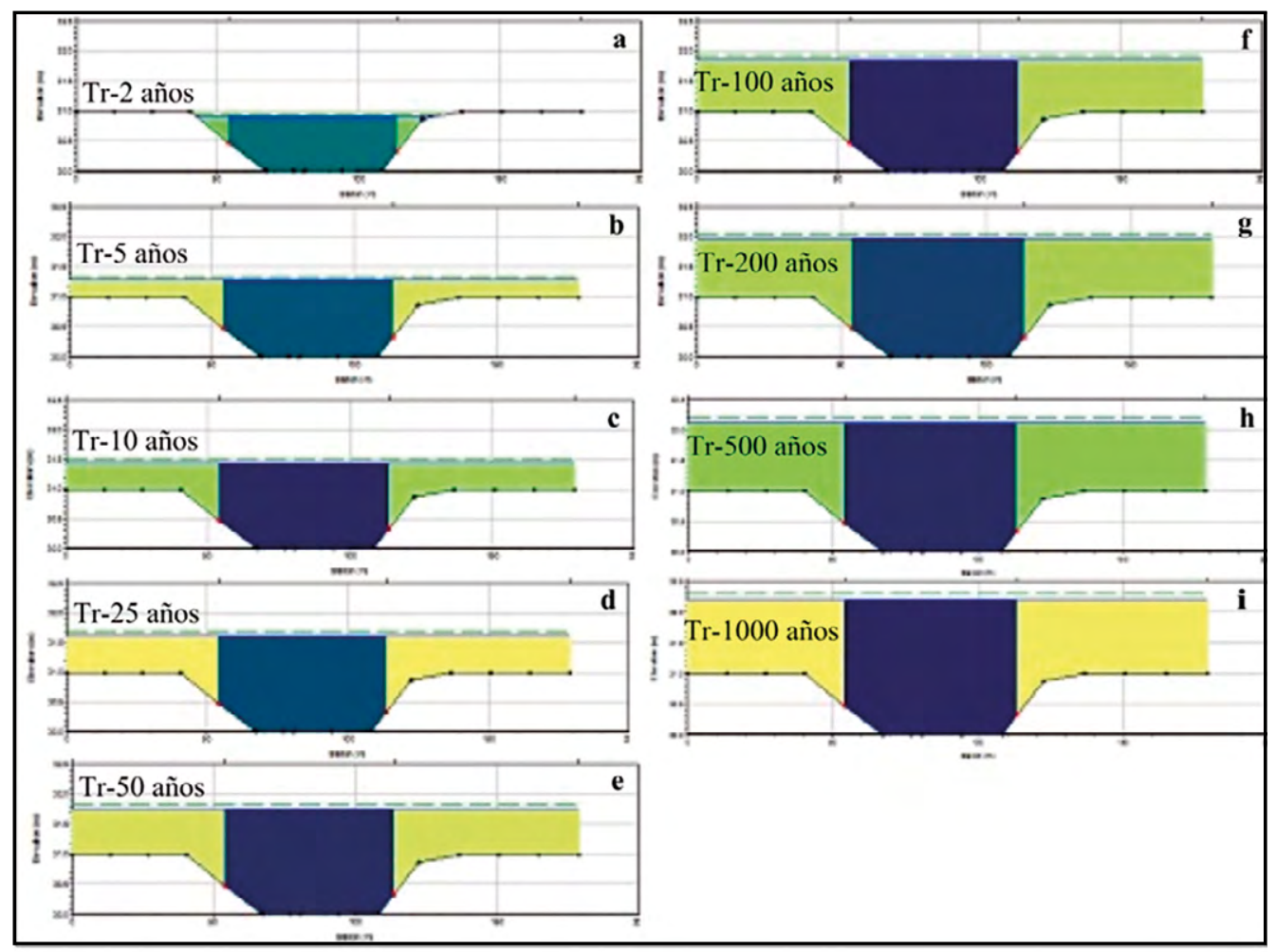

Figura 10. Perfiles de la sección 3072, para los periodos de retorno de 2, 5, 10, 25, 50, 100, 200,500 y 1000 años; su ubicación en planta se muestra en la figura 10. En el eje de las abscisas se indica la amplitud del cauce o canal $(80 \mathrm{~m}$ ) y del valle aluvial (50 $\mathrm{m}$ a cada lado) y en el de las ordenadas la profundidad del canal ( $1 \mathrm{~m}$ ), tirante de agua y la altura del espejo de agua que desborda del cauce del arroyo hacia su valle aluvial (zona de influencia). Los colores indican la distribución de velocidad del agua. Se indican las máximas velocidades con las tonalidades más intensas.

Elaboración propia.

Para lluvias asociadas a un periodo de retorno de 10 años, descritas anteriormente como similares a las del huracán Jimena en 2009 (figura 9) y de acuerdo con la información de la sección 10c, el tirante de agua es de $1.45 \mathrm{~m}$ (tabla 3), por lo que su valle aluvial es inundado con un espejo de agua de $0.45 \mathrm{~m}$, consistente con la información colectada con habitantes de Ciudad Insurgentes, quienes refieren al huracán Jimena como el que mayores daños económicos ha causado (figura 11 [b]). Considerando esto con la información de las tablas 2 y 3 , así como la de las figuras 9 y 10 , sería de esperar que ante lluvias asociadas a periodos de retorno de 25 y 50 años, las pérdidas económicas en el poblado aumentaran considerablemente (figuras 10 [d] y [e]) $y$ ante escenarios por inundaciones fluviales para periodos de retorno de 100, 200, 500 y 1000 años, adquirirían dimensiones de desastres (figuras 10 [f], [g], [h], [i]). Para validar los resultados del modelaje unidimensional los autores del presente trabajo retomaron la información de la figura 9, consistente con la de áreas vulnerables a inundaciones fluviales en Ciudad Insurgentes del Atlas de Riesgos Naturales de Comondú, que se muestra en la figura 7.

De igual manera y siguiendo la metodología del CENAPRED (CENAPRED, 2013; Ramos Radilla, 2006; Salas Salinas, 2011 ) se emplea para validar el modelaje la información colectada en campo, con habitantes de Ciudad Insurgentes, quienes proporcionaron evidencias fotográficas como las que se muestran en la figura 11 (a) para el huracán Norbert en 2008 y figura 11 (b), para el huracán Jimena en 2009, cuya inundación fue generada por un espejo de agua de $0.25 \mathrm{~m}$ y $0.45 \mathrm{~m}$, equivalentes al modelaje para periodos de retorno de 5 y 10 años, respectivamente (figura 10 [b] y [c]). Es posible asumir que el modelaje efectuado brinda una buena aproximación de los posibles escenarios de inundación esperados, según la estimación de los periodos de retorno para lluvias intensas. 


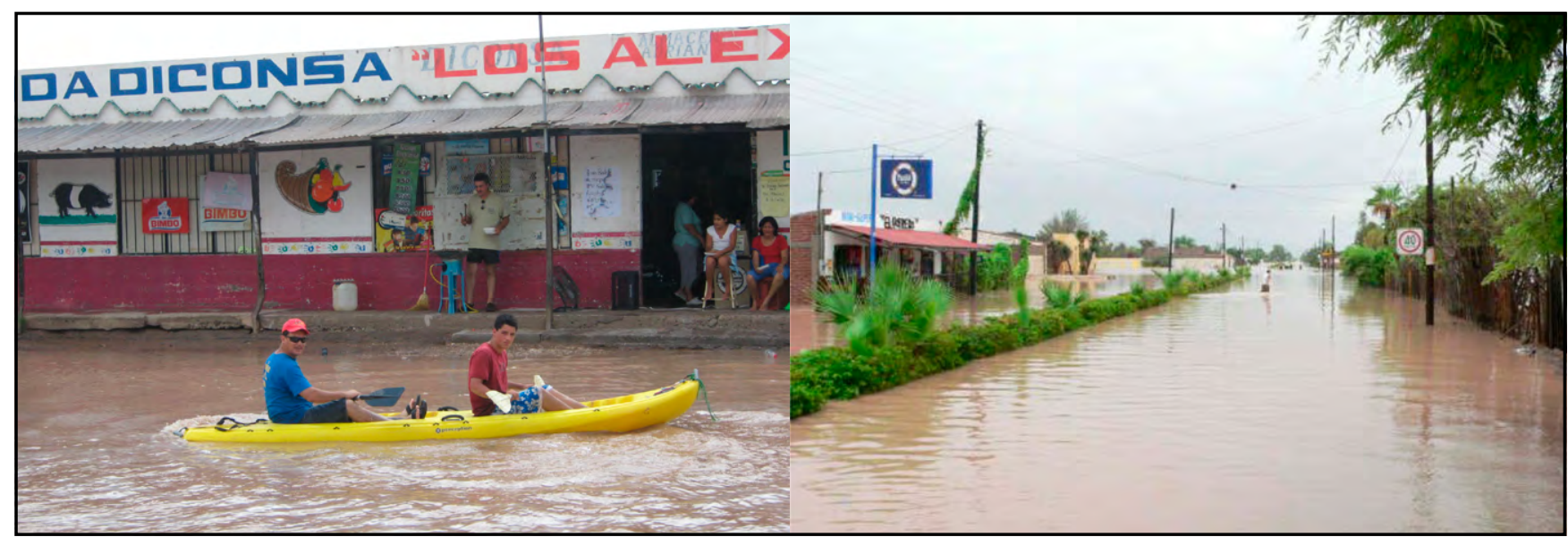

Figura 11. a) Inundación de Ciudad Insurgentes por lluvias intensas al paso del huracán Norbert en octubre de 2008. El espejo de agua que se observa posterior al paso del huracán es de entre 0.20 y 0.25 m; b) Bulevar Emiliano Zapata inhabilitado por la inundación provocada por el desbordamiento del arroyo Insurgentes debido a las lluvias intensas al paso del huracán Jimena en septiembre de 2009. El espejo de agua que se observa posterior al paso del huracán es de entre 0.40 y $0.50 \mathrm{~m}$.

Fotografías proporcionadas por Rubén González González.

\section{DISCUSIÓN}

Con la evolución de los medios de comunicación, que permiten enterarse en tiempo real de lo que ocurre al otro lado del mundo, se ha creado una percepción generalizada en el aumento de desastres. De acuerdo con Wijkman y Timberlake (1985, p.31),

aun cuando las fuerzas naturales desempeñan un papel importante en la iniciación de multitud de desastres, ya no deben seguir considerándose como la causa principal de los mismos. Tres parecen ser las causas fundamentales que dominan los procesos de desastre en el mundo en desarrollo, que es, precisamente, donde su incidencia es mayor:

1) La vulnerabilidad humana, resultante de la pobreza y la desigualdad

2) La degradación ambiental resultante del abuso de las tierras

3) El rápido crecimiento demográfico

El riesgo de desastres puede asumirse como la suma de los procesos de origen humano que interactúan con una amplia gama de procesos naturales (Chávez López, 2017). De acuerdo con el análisis y descripción de los aspectos geomorfológicos y del patrón fluvial de la cuenca del arroyo Insurgentes, el problema de inundaciones en el poblado se presenta a partir del año 2008, cuando se registra un incremento demográfico y con este del área urbana, que invade los cauces del arroyo Insurgentes y el de su tributario de primer orden. Ello puede referirse como degradación ambiental, resultante del abuso de las tierras, que se acentúa con la deforestación de terrenos para la actividad agrícola. La actividad humana se incorpora como factor inducido (antrópico), en el entorno geomorfológico de la cuenca, que se refleja como pérdida de área de escurrimiento. Ante eventos de lluvias extraordinarias asociadas generalmente a la incidencia aleatoria de fenómenos meteorológicos se ha ido incrementando la vulnerabilidad de los habitantes de Ciudad Insurgentes a los efectos causados por las inundaciones.

Situaciones parecidas se presentan en las principales ciudades del estado de Baja California Sur; sin embargo, la información publicada es escasa, como la referente al poblado de San José del Cabo, en donde el entorno geomorfológico de la parte baja de su cuenca ha sido modificado por la actividad turística (Chávez López \& Mendoza Albañez, 2016) y la publicada por Wurl, Martínez García e Imaz Lamadrid (2014), para el oasis La Purísima, en donde el poblado del mismo nombre se encuentra en una cañada. En ambos casos las inundaciones se presentan como pérdida de área de escurrimiento debido al incremento del área urbana, en donde el factor común son los cambios indiscriminados de uso del suelo, asentamientos irregulares (invasiones) en zonas consideradas de alto riesgo y la indiferencia de los gobiernos, tanto municipal, como estatal y federal, ante estas situaciones, hasta que ocurre un desastre. También es de destacar el hecho de que aun con la tendencia del llamado desarrollo sustentable, se continúa con la 
filosofía de adaptar el medio ambiente a nuestras necesidades e intereses; aspectos que no son privativos del estado de Baja California Sur, ya que situaciones muy parecidas se presentan en todo México y en los países de economías emergentes.

Si bien el presente trabajo no tiene el alcance de uno de gestión de riesgos (multidisciplinario), el diagnóstico de los aspectos físico-ambientales de la cuenca, identificación de amenazas (frecuencia, magnitud, intensidad) y elaboración de escenarios de peligro, son aspectos básicos para la gestión no sólo de riesgo, ya que la información generada es de utilidad para los programas de planeación y ordenamiento territorial.

\section{CONCLUSIONES}

Se encontró que los aspectos geomorfológicos y morfométricos de la cuenca del arroyo Insurgentes, así como su aspecto climático, que se refleja en la aridez de la región, no son factores que incrementen el riesgo a inundaciones en el poblado de Ciudad Insurgentes. Los problemas de inundaciones se presentan asociados a lluvias intensas ocasionadas por fenómenos meteorológicos y por el incremento del área urbana que invade los cauces del arroyo Insurgentes. Esto último refleja la falta de planeación y ordenamiento en el incremento de su área urbana, por lo que el peligro de inundaciones en Ciudad Insurgentes debe considerarse como un riesgo inducido por la actividad humana.

Puesto que la construcción de los posibles escenarios de peligro por inundaciones se asume como la probabilidad de ocurrencia de un fenómeno potencialmente dañino sobre el medio natural y humano en su área de influencia, encontramos que el área inundable, para un periodo de retorno de 1000 años, puede llegar a incrementarse en $94 \%$ con respecto al periodo de retorno de 10 años. El poblado de Ciudad Insurgentes puede alcanzar situaciones críticas de inundaciones.

Por la ubicación geográfica de Ciudad Insurgentes, es evidente que no se puede eliminar el riesgo de inundaciones, pero sí reducir sus efectos planificando el incremento de su área urbana. Es fundamental para los gobiernos estatal y municipal un proceso de toma de decisiones frente a la incertidumbre que genera la condición aleatoria de los fenómenos meteorológicos, considerando los periodos de retorno de 100, 200, 500 y 1000 años, como la probabilidad de ocurrencia de lluvias máximas esperadas, para el diseño de obras de protección con un buen margen de confiabilidad y reducir el riesgo de inundaciones que garantice la integridad de la población.

\section{REFERENCIAS}

- Arriaga, L., Coria, R., \& Bastida-Zavala, R. (Eds.). (1992). La Sierra de La Laguna, Fundamentos para apoyar la creación de una Reserva de la Biósfera. Publicación No. 7. La Paz, B. C. S., México: Centro de Investigaciones Biológicas del Noroeste, S. C.

- Centro Nacional de Prevención de Desastres. (2009). Características e impacto socioeconómico de los principales desastres ocurridos en la República mexicana en el año 2008 [Ficha bibliográfica, índice y pdf descargable]. Recuperado de http://www.cridlac.org/digitalizacion/pdf/spa/doc17830/ doc17830-contenido.pdf

(2013). Guía de Contenido Mínimo para la Elaboración de Atlas Estatales y Municipales de Peligros y Riesgos Versión 2013 [Listado]. Distrito Federal, México: Autor.

- Chávez López, S. (2017). ¿Cuándo empezamos a definir y conceptualizar el riesgo? Recursos Naturales y Sociedad, 3(1), 19-27. Recuperado de https://www.cibnor.gob.mx/revista-rns/ pdfs/vol3numl/RE-al7completo.pdf
- Chávez López, S., \& Mendoza Albañez, A. (2016). Aspectos geomorfológicos e hidrográficos de la cuenca de San José del Cabo, Baja California Sur, México, como factor de riesgo a inundaciones. Áreas Naturales Protegidas SCRIPTA, 2(2), $103-$ 126.

- Comisión Nacional del Agua. (20 de abril de 2015). Determinación de la disponibilidad de agua en el acuífero Santo Domingo estado de Baja California Sur, México. Diario Oficial de la Federación. Recuperado de https://www.gob.

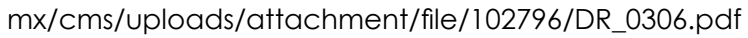

- García Gutiérrez, L. M. (2003). Glosario de términos geológicos. Pachuca, México: Consejo de Recursos Minerales y Asociación de Ingenieros de Minas, Metalurgias y Geólogos de México, A. c.

- Gobierno del estado de Baja California Sur. (2015). Baja California Sur Información Estratégica [Documento informativo]. Recuperado de http://www.spyde.bcs.gob.mx/ files/die/2015/EstrategicoBCSJulio2015.pdf 
- Gutiérrez Elorza, M. (2008). Geomorfología. España: Prentice Hall.

- H. XIII Ayuntamiento de Comondú. (2010). Estudio para la recuperación del Acuífero del Valle de Santo Domingo. Baja California Sur, México: Municipium.

- H. XIV Ayuntamiento de Comondú. (2011). Atlas de Riesgos Naturales de Comondú, Baja California Sur. México: Municipium.

- Instituto Nacional de Estadística y Geografía. (1988, 2000). Cartas topográficas vectoriales escala 1:50 000. G12C37 Ciudad Insurgentes con fotografía aérea de 1978 y G12C47 Benito Juárez con fotografía aérea de 1993 [Imágenes]. México: Autor.

- Instituto Nacional de Estadística y Geografía. (1996). Estudio Hidrológico del estado de Baja California Sur. Aguascalientes, México: Instituto Nacional de Estadística, Geografía e Informática.

- Instituto Nacional de Estadística y Geografía. (2016a). Censos y Conteos de Población y Vivienda. Recuperado de http:// www.inegi.org.mx/default.aspx

- Instituto Nacional de Estadística y Geografía. (2016b). Simulador de flujos de agua de cuencas hidrográficas SIATL [Aplicación operable]. Recuperado de http://antares.inegi.org.mx/ analisis/red_hidro/siatl/\#

- Instituto Nacional para el Federalismo y el Desarrollo Municipal. (1987). Enciclopedia de los Municipios y Delegaciones de México. 1987-1988. Estado de Baja California Sur. Comondú [Ficha informativa]. Recuperado de http://siglo.inafed.gob.mx/ enciclopedia/EMM03bajacaliforniasur/municipios/03001a. html

- López Ramos, E. (1979). Geología de México. Tomo II (2a. ed.). Distrito Federal, México: Universidad Nacional Autónoma de México.
- Lugo Hubp, J. (1989). Diccionario geomorfológico. Distrito Federal, México: Universidad Nacional Autónoma de México.

- National Oceanic Atmospheric Administration. (2016). Archives, Other archives: HURDAT, track maps, marine products and more [Base de datos]. Recuperado de http://www.nhc.noaa. gov/data/

- Ramos Radilla, V. (Coord.). (2006). Guía básica para la elaboración de atlas estatales y municipales de peligros y riesgos: Fenómenos hidrometeorológicos. En serie Atlas Nacional de Riesgos. México: CENAPRED. Recuperado de http://www. proteccioncivil.gob.mx/work/models/ProteccionCivil/Resource/375/1/images/fh.pdf

- Salas Salinas, M. E. (2011). Metodología para la elaboración de mapas de riesgo por inundaciones en zonas urbanas. En serie Atlas Nacional de Riesgos. México: CENAPRED. Recuperado de http://www.cenapred.gob.mx/es/Publicaciones/archivos/203.pdf

- Silva Machorro, C. (1978). Unidades del suelo: Interpretadas para su uso en ingeniería civil y aprovechadas por el campesino en usos agropecuarios. (2a ${ }^{a}$ ed.). México: Continental.

- Troyo-Diéguez, E., Cruz-Falcón, A., Norzagaray-Campos, M., Beltrán-Morales, L. F., Murillo-Amador, B., Beltrán-Morales, F. A., ..., Valdez-Cepeda, R. D. (2010). Agotamiento hidro-agrícola a partir de la Revolución Verde: Extracción de agua y gestión de la tecnología de riego en Baja California Sur, México. Estudios Sociales, 18(36), 179-201.

- Wijkman, A., \& Timberlake, L. (1985). Desastres naturales ¿̇Fuerza mayor u obra del hombre? Washington, DC: Instituto Internacional por el Medio Ambiente y el Desarrollo.

- Wurl, J., Martínez García, C. N., \& Imaz Lamadrid, M. A. (2014). Caracterización del peligro por inundaciones en el oasis La Purísima, Baja California Sur, México. Investigaciones Geográficas, Boletín del Instituto de Geografía, 87, 76-87. doi: 10.14350/ rig. 41858 